\title{
Intraoperative ventricular volume restoration by intraventricular Ringer solution injection in a normal-pressure hydrocephalus patient with traumatic bilateral acute subdural hematoma and ventricular system collapse caused by cerebrospinal fluid shunt overdrainage: illustrative case
}

\author{
Dalibor Sila, MD, ${ }^{1}$ Karim Morsi, MD, ${ }^{1}$ Markus Lenski, MD, ${ }^{2}$ and Stefan Rath, $M D^{1}$ \\ ${ }^{1}$ Department of Neurosurgery and Interventional Neuroradiology, Donau-Isar Klinikum, Deggendorf, Germany; and ${ }^{2}$ Neurosurgical Clinic, Clinic of the University of Munich \\ (LMU), Campus Grosshadern, Munich, Germany
}

\begin{abstract}
BACKGROUND The authors report a case of a patient with normal-pressure hydrocephalus treated with a ventriculoperitoneal shunt who developed a traumatic hemispheric bilateral acute subdural hematoma caused by quick cerebrospinal fluid (CSF) overdrainage. The authors present active ventricular CSF volume restoration as a novel treatment option. CSF overdrainage in patients with shunts may facilitate acute subdural hematoma formation even in cases of minor head trauma. Therapeutic options include CSF shunt function restriction or ligation, hematoma evacuation, or a combination of both.

OBSERVATIONS In this case, the authors performed emergency surgery with hematoma evacuation through a bilateral craniotomy and actively restored the volume of the ventricular system with a slow intraventricular injection of $37^{\circ} \mathrm{C}$ warmed Ringer solution through a shunt burr hole reservoir.

LESSONS In addition to hematoma evacuation and restriction of shunt function, the intraoperative restoration of ventricular volume could be a treatment option to prevent postoperative rebleeding or a space-occupying air collection in the subdural space. The risk of possible complications, such as ventricular or parenchymal bleeding, shunt dysfunction, or infections, requires further investigation. Restoration of the ventricular CSF volume by intraventricular injection of Ringer solution was in this case an efficient treatment method to prevent subdural rebleeding and a space-occupying air collection after subdural hematoma evacuation.
\end{abstract}

https://thejns.org/doi/abs/10.3171/CASE21188

KEYWORDS acute subdural hematoma; CSF overdrainage; ventricular CSF volume restoration; ventriculoperitoneal shunt; hydrocephalus

Cerebrospinal fluid (CSF) overdrainage is a common complication of ventriculoperitoneal shunt devices. ${ }^{1}$ Acute subdural hematomas are of particular concern in patients with normal-pressure hydrocephalus treated with CSF shunting because even minor head trauma may result in subdural hematoma formation. The presence of a ventricular shunt facilitates further expansion of the subdural hematoma and often necessitates surgical treatment, including subdural hematoma evacuation and shunt ligation. ${ }^{2}$ The treatment options are potentially heterogeneous, but in the case of a spaceoccupying acute subdural hematoma with the risk of brain herniation, emergency surgery and hematoma evacuation are necessary.
In this technical case report, we summarize the therapeutic strategy mentioned in the literature and present active ventricular CSF volume restoration as a novel treatment option.

\section{Illustrative Case}

History and Examination

A 66-year-old woman with a history of normal-pressure hydrocephalus treated with a ventriculoperitoneal shunt (CODMAN CERTAS, DePuy Synthes), pressure level 2, and gait disturbance without further focal neurological deficits and no history of anticoagulation therapy

ABBREVIATIONS CSF $=$ cerebrospinal fluid; $C T=$ computed tomography. INCLUDE WHEN CITING Published May 31, 2021; DOI: 10.3171/CASE21188. SUBMITTED March 30, 2021. ACCEPTED April 7, 2021.

(c) 2021 The authors, CC BY-NC-ND 4.0 (http://creativecommons.org/licenses/by-nc-nd/4.0/). 

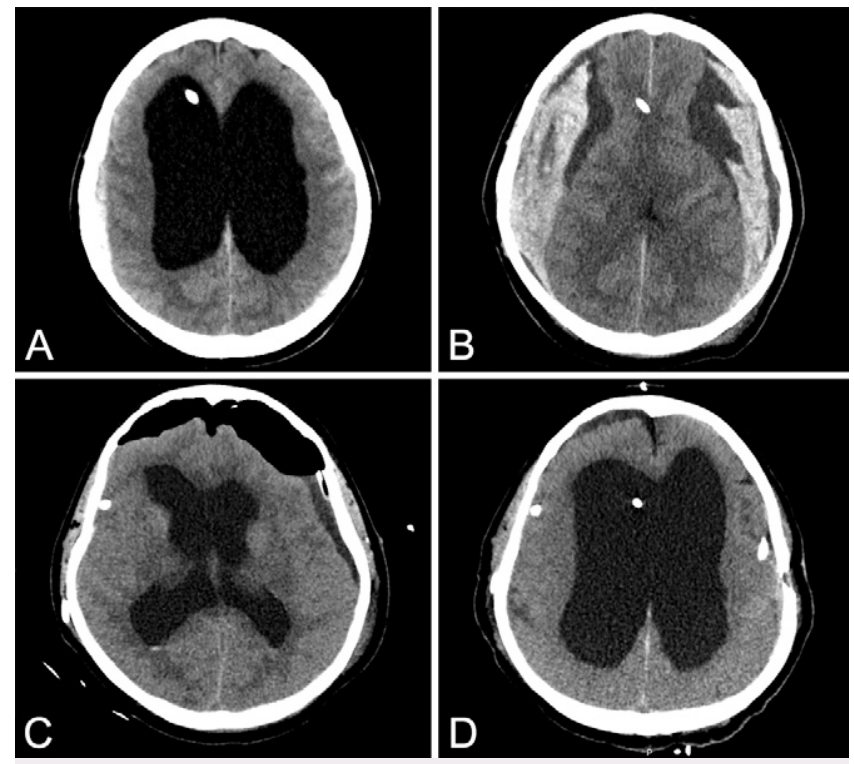

FIG. 1. Initial CT scan showing bilateral narrow acute subdural hematomas (A). CT scan after 4 hours showing expansion of subdural hematomas caused by CSF overdrainage (B). CT scan on day 1 after surgery (C). CT scan on day 4 after surgery (D).

was admitted to a district hospital after a fall from a ladder. After she experienced a cerebral seizure, cranial computed tomography (CT) (Fig. 1A) was performed and confirmed acute narrow bilateral parietal subdural hematomas and decent left-sided traumatic subarachnoid hemorrhage. Because of rapid neurological deterioration (Glasgow Coma Scale score 3), intubation and transport to our neurosurgical department were necessary. At the time of admission, the patient was sedated, ventilated, and received catecholamine circulation support. Her pupils were isocoric with delayed light reaction. A control CT scan obtained 4 hours after the initial one (Fig. 1B) showed large hemispheric bilateral acute subdural hematomas with mass effect and compression of both lateral ventricles but without midline shift. We



FIG. 2. Intraoperative photograph at the beginning of ventricular injection, with burr hole reservoir puncture and shunt catheter clipping (arrows). decided on immediate transport to the operating room and surgical evacuation of the hematomas.

\section{Technical Details}

Surgery was performed with the patient under intravenous general anesthesia. A central venous catheter and an invasive blood pressure monitor were inserted. Ringer solution was warmed to $37^{\circ} \mathrm{C}$. After head shaving and skin disinfection, an incision $12 \mathrm{~cm}$ in length was made in the coronary plane on both sides, and bleeding was stopped with bipolar coagulation and with a setting on ScalpFix clips (Aesculap). Identification and temporary clipping of the shunt catheter with a coated clamp (Fig. 2) were necessary. Afterward, frontoparietal craniotomy of approximately $9 \mathrm{~cm}$ in diameter was performed on both sides. After overlaying the craniotomy edges with cotton pads and making a dural incision, we evacuated the hematomas from the subdural space on both sides with suction and catch tongs. Under direct view, the surgeon restored the volume of the collapsed ventricular system by injecting $340 \mathrm{ml}$ of $37^{\circ} \mathrm{C}$ warmed Ringer solution over the puncture of the shunt burr hole reservoir for a period of 30 minutes (Fig. 2) until the brain expanded slowly. The filling volume was calculated according to the volume of the lateral ventricles observed on the initial head CT scan using the formula $A \times B \times C / 2$. Afterward, we placed subdural drains on both sides and sutured the dura. Over a small supraclavicular incision, we ligated the shunt catheter with the silk ligature and removed the temporary catheter clamp. At the end, the bone was fixated with plates, suction drains were placed in the subgaleal position on both sides, and the skin was sutured. After the surgery, the patient was transported to the intensive care unit.

\section{Follow-Up}

Sedation and catecholamine support reduction were performed on day 1 after surgery. On the control CT scan on day 1 , residual subdural air and hygroma were seen, and the ventricular volume was restored (Fig. 1C). On day 2, we were able to extubate the patient with a sufficient level of consciousness; she demonstrated a slight palsy of the left arm as well as dysphagia. On day 4, we performed CT again (Fig. 1D), and afterward the subdural drains as well as the supraclavicular silk shunt catheter ligation were removed under a local anesthetic. Shunt (CODMAN CERTAS) pressure was adjusted to level 6 . Because of dysphagia, aspiration, and development of respiratory failure, intubation and bronchoscopy were necessary. On day 7 , the shunt pressure level was adjusted from level 6 to 4 . On day 10, the patient was extubated again. On day 17, we transferred the patient to the intermediate care unit, and on day 26 , the patient was released into rehabilitation. During rehabilitation, the patient's neurological deficit improved partially. The patient was mobile, but she presented with gait disturbance and slight psychomotor delay. CT performed after rehabilitation demonstrated a regular result without rebleeding or chronic subdural hematoma formation.

\section{Discussion}

The therapy for subdural hematoma in patients with shunts is extremely challenging because of heterogeneous treatment options such as programmable shunt valve function restriction, shunt ligation, hematoma evacuation, and a combination of these techniques. Programmable shunt valves include a nonoperative treatment option for conservative management of acute subdural hematoma in patients with ventricular shunts. Increasing the shunt valve open 
pressure may result in rapid resolution of the acute subdural hematoma in some patients. ${ }^{2}$ However, increased shunt valve restriction as well as shunt catheter ligation can lead to hydrocephalus formation and clinical deterioration in shunt-dependent patients. The therapy by shunt ligation requires secondary ligation removal and represents a risk of infection. Berger et al. analyzed eight cases of acute subdural hematoma treatment in patients with CSF shunting and reported improvement after resetting the shunt valve to maximal pressure in two cases, but in six cases, surgical treatment was necessary. ${ }^{3}$ Five of them were also primarily treated by resetting the shunt valve. In our case, the alternative to shunt tubing ligation could be resetting of the shunt valve. The CODMAN CERTAS valve setting of 8 is a virtual "off" setting. However, the valve adjustment tool was not available during the emergency surgery.

\section{Observations}

In the case of mass effect of the subdural hematoma and rapid neurological deterioration of the patient, emergency surgery is necessary. Hematoma removal alone may result in hematoma recurrence and may require a second treatment comprising shunt management to effectively control the hematoma. ${ }^{4}$ In this case, the ventricular system collapse was the cause of large bilateral subdural spaces after hematoma evacuation, which means a high risk of postoperative subdural rebleeding or space-occupying air collection. To prevent these complications, we reduced the subdural space after restriction of the shunt function by additional restoration of the ventricular CSF volume through slow intraventricular injection of warmed Ringer solution via a shunt burr hole reservoir. At the end of ventricular injection, the brain volume was nearly normal, but frontally we still saw a little free subdural space. Because of a lack of experience with this treatment method, we did not want to risk bleeding complications by additional ventricular filling. That is why the frontal residual subdural air collection was observed on the day 1 CT scan (Fig. 1C).

\section{Lessons}

For the first time, to our knowledge, we report restoration of the ventricular CSF volume by slow intraventricular injection of warmed Ringer solution as an efficient treatment method to prevent subdural rebleeding and space-occupying air collection after subdural hematoma evacuation in patients with shunts. The active reconstruction of a collapsed ventricular system, particularly in patients with extensive internal hydrocephalus, could be the main factor preventing acute rebleeding, especially in the early stage after the surgery. At the same time, shunt function restriction is an important step in preventing subacute subdural bleeding with possible transition to a chronic subdural hematoma. In our opinion, this technique can be useful in cases of a large subdural space after hematoma evacuation. However, the risk of possible complications such as ventricular or cerebral hemorrhage caused by quick injection, secondary shunt dysfunction, or CSF infections should be considered. The risk of late complications after shunt reopening, such as subacute rebleeding with successive chronic subdural hematoma formation, should be considered. Therefore, delayed control CT should be performed. The effect of this therapeutic strategy compared with the discussed treatment options deserves further expert investigation.

\section{References}

1. Noleto G, Neville IS, Tavares WM, et al. Giant acute epidural hematoma after ventriculoperitoneal shunt: a case report and literature review. Int J Clin Exp Med. 2014;7(8):2355-2359.

2. Hayes J, Roguski M, Riesenburger RI. Rapid resolution of an acute subdural hematoma by increasing the shunt valve pressure in a $63-$ year-old man with normal-pressure hydrocephalus with a ventriculoperitoneal shunt: a case report and literature review. J Med Case Rep. 2012;6:393.

3. Berger A, Constantini S, Ram Z, Roth J. Acute subdural hematomas in shunted normal-pressure hydrocephalus patients-management options and literature review: a case-based series. Surg Neurol Int. 2018;9:238.

4. Hoya K, Tanaka Y, Uchida T, et al. Treatment of traumatic acute subdural hematoma in adult hydrocephalus patients with cerebrospinal fluid shunt. Clin Neurol Neurosurg. 2012;114(3):211-216.

\section{Disclosures}

The authors report no conflict of interest concerning the materials or methods used in this study or the findings specified in this paper.

\section{Author Contributions}

Conception and design: Sila, Lenski, Rath. Acquisition of data: Morsi. Analysis and interpretation of data: Lenski. Drafting the article: Sila, Lenski, Rath. Critically revising the article: Morsi, Lenski, Rath. Reviewed submitted version of manuscript: Lenski, Rath. Approved the final version of the manuscript on behalf of all authors: Sila. Administrative/technical/material support: Morsi, Lenski. Study supervision: Lenski, Rath.

\section{Correspondence}

Dalibor Sila: Donau-Isar Klinikum, Deggendorf, Germany. dalibor.sila@ donau-isar-klinikum.de. 